\title{
Cisplatin-induced alterations in the blood-nerve barrier: effects of combination of vitamin B1, B6 and B12
}

\author{
A. Tothonglor, P. Kobutree, A. Roumwong, D. Jindatip, S. Agthong \\ Department of Anatomy, Faculty of Medicine, Chulalongkorn University, Pathumwan, Bangkok, Thailand
}

[Received: 24 December 2021; Accepted: 9 January 2022; Early publication date: 21 January 2022]

Background: Cisplatin is a chemotherapeutic agent against solid cancers. However, neuropathy is a major side effect and has no effective treatment so far. Emerging evidence suggests that cisplatin might damage nerve capillaries leading to impaired blood-nerve barrier (BNB). This study aimed to investigate the ultrastructural changes of the BNB in the sciatic nerves and dorsal root ganglia of rats with cisplatin neuropathy and the effects of $B_{1-6-12}$.

Materials and methods and Results: The results showed that cisplatin $2 \mathrm{mg} / \mathrm{kg}$ injected intraperitoneally twice a week for 5 consecutive weeks caused thermal hypoalgesia and structural abnormalities of nerves and ganglia. Co-treatment with oral $B_{1-6-12}(100: 100: 1) 100,300$ and $600 \mathrm{mg} / \mathrm{kg} /$ day for 5 weeks reduced the sensory deficit and structural alterations. Electron microscopic analysis demonstrated the higher frequencies and wider distances of pericyte detachment in the capillaries of cisplatin than control groups. Vitamin B1, B6 and B12 especially the medium dose, reversed these abnormalities. Culture of endothelial cells and pericytes with cisplatin demonstrated reduced cell viability, increased caspase-3 activity, lower transendothelial electrical resistance and decreased expression of tight junction proteins, occludin and zonula occluden-2.

Conclusions: Vitamin B1, B6 and B12 could correct these toxic effects of cisplatin. These data confirm that cisplatin causes pathological alterations in the components of $B N B$ which correlate with the severity of neuropathy. Furthermore, $B_{1-6-12}$ is effective against these abnormalities and deserves further investigations as potential treatment for cisplatin-induced neuropathy. (Folia Morphol 2023; 82, 1: 53-62)

Key words: pericyte, endothelial cell, cisplatin, nerve, neuropathy

\section{INTRODUCTION}

Cisplatin is an antineoplastic agent used to treat cancers of various organs [6]. One of its major side effects is peripheral neuropathy often leading to dose reduction or cessation and thus effectiveness of chemotherapy. Cisplatin-induced neuropathy is mainly characterised by sensory abnormalities in both animal models and patients $[2,16,22,28]$. Pathological examination showed degeneration and demyelination of nerve fibres [1, 29]. Although various underlying mechanisms have been identified and relevant agents were tested, no clinically effective drugs were achieved so far $[18,24]$.

Address for correspondence: Dr. S. Agthong, Department of Anatomy, Faculty of Medicine, Chulalongkorn University, 1873 Rama IV Road, Pathumwan, 10330 Bangkok, Thailand, e-mail: sagthong@hotmail.com

This article is available in open access under Creative Common Attribution-Non-Commercial-No Derivatives 4.0 International (CC BY-NC-ND 4.0) license, allowing to download articles and share them with others as long as they credit the authors and the publisher, but without permission to change them in any way or use them commercially. 
Accumulating evidence suggests vascular dysfunction as another potential mechanism. Patients receiving cisplatin-based chemotherapy had arterial occlusion [17] and endothelial damage [5]. In cisplatin-treated rats, reduced nerve blood flow, decreased number of vasa nervorum and endothelial apoptosis were reported [15]. Recently, the lower density and detachment of nerve pericytes have been found in the rats with cisplatin neuropathy [12]. These abnormalities might impair blood-nerve barrier (BNB). This study aimed to further clarify the association of cisplatin neuropathy with BNB defects. In addition, since beneficial effects of vitamin $B 1, B 6$ and $B 12$ in combination $\left(B_{1-6-12}\right)$ were seen in the preliminary study, whether this improvement in neuropathy was associated with ameliorated BNB abnormalities was also investigated.

\section{MATERIALS AND METHODS}

\section{Animals}

The experiment was approved by the institutional ethics committee (Ref. No. 19/58) and carried out in accordance with the Animals for Scientific Purposes Act 2015, Thailand. Thirty female Wistar rats weighing $250 \mathrm{~g}$ were divided into five groups: control (C), cisplatin $(\mathrm{P})$, cisplatin + low-dose $(\mathrm{P}+\mathrm{LB})$, medium-dose $(\mathrm{P}+\mathrm{MB})$ or high-dose $\mathrm{B}_{1-6-12}(\mathrm{P}+\mathrm{HB})(\mathrm{n}=6$ for each group).

\section{Drug administration}

The cisplatin and cisplatin $+B_{1-6-12}$ groups received cisplatin (Pfizer, USA) diluted in normal saline to the final concentration of $0.5 \mathrm{mg} / \mathrm{mL}$ for intraperitoneal injection. The dose of cisplatin was $2 \mathrm{mg} / \mathrm{kg}$ twice a week for 5 continuous weeks ( $20 \mathrm{mg} / \mathrm{kg}$ cumulative dose). This dose regimen has been shown to induce peripheral neuropathy in rats $[2,28]$. The control group received normal saline injection with the volume and schedule equivalent to the cisplatin groups. B1, B6 and B12 (all from Sigma) (100:100:1 by weight) were dissolved in normal saline and given by gavage during the cisplatin treatment once daily for 5 weeks. This ratio of $B_{1-6-12}$ was selected based on the previous studies $[3,13]$. Low-dose, medium-dose and high-dose $B_{1-6-12}$ groups received 100, 300 and $600 \mathrm{mg} / \mathrm{kg} /$ day, respectively.

\section{Hind-paw thermal nociception}

The details of procedure are described elsewhere [29]. Briefly, the test was done at baseline and the end of $3^{\text {rd }}$ and $5^{\text {th }}$ weeks. Each rat was placed on the hot plate analgesia meter (Harvard Apparatus, UK) maintained at $55^{\circ} \mathrm{C}$. When the rat licked its hind paw on either side, elapsed time was recorded as latency. The cut-off duration of $35 \mathrm{~s}$ was set to avoid skin burn. The test was repeated at least 3 times with an interval of $15 \mathrm{~min}$ and the mean latency was obtained for each rat.

\section{Tissue collection}

After the last injection of cisplatin, all the rats were sacrificed by overdose anaesthetics and then transcardially perfused with normal saline. This was followed by $3 \%$ glutaraldehyde. L4,5 dorsal root ganglion (DRG) with the proximal and distal parts of sciatic nerves (divided at the trifurcation) were removed, post-fixed in 3\% glutaraldehyde and embedded in epoxy resin. These specimens were used for morphometric analysis.

\section{Nerve morphometry}

Transverse $1 \mu \mathrm{m}$-thick sections of the sciatic nerve were cut, mounted on slides, and stained with paraphenylenediamine. The sections were examined under a light microscope and the cross-sectional areas were chosen using the three-window sampling method [4]. Briefly, under $40 \times$ objective lens, three windows of $0.012 \mathrm{~mm}^{2}$ were randomly placed, one in the middle and the other two in the periphery of fascicle. Images of these windows were imported into a computer via a digital camera. Morphometric analysis was done to obtain the number of myelinated fibres, axon diameter, myelinated fibre density, myelin thickness and $g$ ratio using the Image-Pro Plus software ${ }^{\circledast}$. The values derived from the three windows were extrapolated to the whole nerve.

\section{DRG morphometry}

The L4 DRG were serially cut into $2 \mu \mathrm{m}$-thick sections and stained with toluidine blue. The estimation for total number of neurons in each ganglion was done using the physical dissector method. Details of the procedures were described elsewhere [28]. In brief, every $20^{\text {th }}$ section was selected and the number of neurons with prominent nucleus and nucleolus was counted. Then, this number was extrapolated to the total number for the whole DRG. In addition, at least 300 neurons in each DRG were randomly analysed for areas of the nucleus and nucleolus using the Image-Pro Plus software. 


\section{Transmission electron microscopy}

Ultrathin sections (70 $\mathrm{nm}$ thickness) of the L5 DRG and sciatic nerves were stained with lead citrate and uranyl acetate. Morphology of pericytes and the basement membrane shared with endothelial cells was observed with a transmission electron microscope (JEM-1400PLUS, Japan). In each rat, 20 capillaries were randomly chosen from serial sections of each tissue (DRG, proximal and distal sciatic nerves). Each capillary was evaluated for the presence of pericyte detachment from endothelial cell and vascular basement membrane (VBM) which was classified into two categories: Category 1 pericyte completely attached to the VBM and endothelial cell, category 2 pericyte detached from the VBM and endothelial cell at some points. Then, the distances at the widest detachment between the pericytes and VBM were measured in the capillaries of category 2 . In addition, the thickness of VBM at the widest separation point was also measured.

\section{Cell culture}

Human umbilical vein endothelial cell (HUVEC) (Invitrogen) and human brain vascular pericyte (HBVP) (ScienCell) were grown according to manufacturers' protocols. Each experiment was performed in triplicate and repeated three times. HUVEC and HBVP were divided into three groups: control, cisplatin, and cisplatin $+B_{1-6-12}$. $B_{1-6-12}$ was prepared in a ratio of 100:100:1 similar to the animal experiment. In cisplatin and cisplatin $+\mathrm{B}_{1-6-12}$ groups, HUVEC and HBVP were incubated with $3 \mu \mathrm{g} / \mathrm{mL}$ and $1.5 \mu \mathrm{g} / \mathrm{mL}$ of cisplatin for 24 hours, respectively. For $B_{1-6-12}$ treatment, the cells were co-incubated with $1 \mu \mathrm{g} / \mathrm{mL}$ of $B_{1-6-12}$ for 24 hours. The above doses were selected according to the preliminary data.

\section{MTT assay}

The MTT (3-(4,5-dimethylthiazol-2-yl)-2,5-diphenyltetrazolium bromide) assay was used to evaluate the viability of HUVEC and HBVP. The cells were seeded at $1 \times 10^{4}$ and $5 \times 10^{3}$ cells/well, respectively, in 96-well plates and allowed to attach for 24 hours. The cells were then treated according to the experimental conditions for 24 hours. Finally, the cells were incubated with $100 \mu \mathrm{L}$ MTT solution (Life technologies, Molecular Probes, USA) for 2 hours. Subsequently, purple formazan crystals were dissolved in $100 \mu \mathrm{L}$ dimethyl sulfoxide. The absorbance was measured at $570 \mathrm{~nm}$ using a microplate reader (Multiskan GO, Thermo Fisher Scientific, USA). The percentage of cell viability was calculated from the mean absorbance of test samples divided by that of negative control.

\section{Caspase-3 assay}

Caspase-3/cpp32 colorimetric assay kit (BioVision, USA) was used to determine the caspase-3 activity. Briefly, the cells were plated at $1 \times 10^{6}$ in the culture vessels. After treatments, the cells were harvested and resuspended in cell lysis buffer. Supernatant of each sample was collected and protein concentration was measured. Then, the sample was diluted with cell lysis buffer to obtain the protein concentration of $1 \mu \mathrm{g} / \mu \mathrm{L}$ and transferred to a 96 -well plate. This was followed by adding working reaction buffer and DEVD-pNA substrate. The plate was incubated at $37^{\circ} \mathrm{C}$ for 2 hours. The absorbance was measured at $405 \mathrm{~nm}$ using a microplate reader (Multiskan GO, Thermo Fisher Scientific, USA). The caspase-3 activity of treated cells was compared with that of controls.

\section{Transendothelial electrical resistance (TEER) study}

Human umbilical vein endothelial cell were cultured on the upper chamber of transwell insert (Merck, USA) which was inserted in the 24-well plate at $1 \times 10^{4}$ cells/well. After the cells were grown to confluency, they were treated according to the experimental conditions for 24 hours. Cell resistance (R) was measured using Millicell electrical resistance apparatus (Millicell ${ }^{\circledR}$ ERS-2, Merck, USA). TEER value was calculated using the formula: TEER value $\left(\Omega \mathrm{cm}^{2}\right)$ $=\left(R_{\text {sample }}-R_{\text {blank }}\right) \times$ membrane area $\left(\mathrm{cm}^{2}\right)$.

\section{Western blot analysis}

Human umbilical vein endothelial cell were seeded $2 \times 10^{6}$ cells $/ \mathrm{mL}$ in a cell culture dish. After being treated according to experimental protocols for 24 hours, the cells were incubated on ice with RIPA lysis buffer (Cell Signaling, USA) containing $1 \times$ protease inhibitor cocktail (Cell Signaling, USA) for $5 \mathrm{~min}$. Subsequently, the cells were scraped, centrifuged and the supernatant collected. Protein concentration of the supernatant was determined using PierceTM BCA protein assay (Thermo Scientific, USA). Briefly, the sample $(1.5 \mu \mathrm{g} / \mu \mathrm{L})$ was mixed with the fluorescent dye ( $4: 1$ ratio) and denatured at $95^{\circ} \mathrm{C}$ for $5 \mathrm{~min}$. The marker, samples, antibody diluent, primary antibody (1:200 $\beta$-actin [Cell Signaling], 1:200 ZO-1 [Invitrogen], 1:200 ZO-2 [Invitrogen], 1:200 claudin-5 [Invitrogen], and 1:200 occludin [Invitrogen]), rabbit secondary conjugate, streptavidin-HRP, and Luminol-peroxide were 
added onto the plate according to the manufacturer's protocol. Subsequently, separation and immunodetection were conducted using WES automated western blotting system (ProteinSimple, USA). Density of digital image was analysed using Compass software (ProteinSimple, USA). Expression of each protein was normalised to that of $\beta$-actin.

\section{Statistical analysis}

One-way ANOVA followed by Tukey's post hoc test was used for comparing the above parameters between the experimental groups. The test was done using SPSS for Windows version 23. Statistically significant differences were considered when $p<0.05$.

\section{RESULTS}

\section{Body weight}

At baseline, the average body weight was similar between groups. However, at the $3^{\text {rd }}$ and $5^{\text {th }}$ weeks, the values of all groups receiving cisplatin were significantly decreased compared with that of the control group (data not shown). Food and water consumption including physical activities were similar between the cisplatin only and cisplatin $+\mathrm{B}_{1-6-12}$ groups. There was no mortality in any group during the experiment.

\section{Hind-paw thermal nociception}

Before the treatment, the latencies were not significantly different between groups (Fig. 1). However, at the $5^{\text {th }}$ week, the latencies of the cisplatin group were significantly longer than that of the control group, indicating thermal hypoalgesia. In addition, the cisplatin $+M B$ and cisplatin $+\mathrm{HB}$ groups had significantly shorter latencies than the cisplatin group and not statistically different from the control group. In contrast, the latency of the cisplatin + LB group was close to that of the cisplatin group and significantly longer than that of the control group.

\section{Nerve morphometry}

At the fifth week, morphometric analysis of the sciatic nerve showed that the fibre diameters of the cisplatin including cisplatin + LB and cisplatin + HB groups were significantly lower than those of the control and cisplatin + MB groups (Table 1). There were no significant differences between the cisplatin, cisplatin + LB and cisplatin + HB groups. Furthermore, the fibre densities of the cisplatin and cisplatin $+\mathrm{HB}$ groups were significantly higher than those of the control and cisplatin $+\mathrm{MB}$ groups. The values of

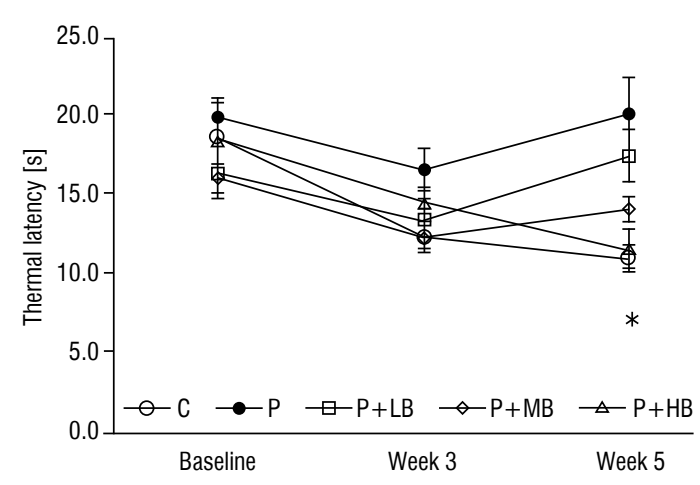

Figure 1. Changes in the thermal latency of hind paw. The graph shows means and standard error of mean; $\mathrm{C}-$ control; $\mathrm{P}-$ cisplatin; $\mathrm{P}+\mathrm{LB}$ - cisplatin + low-dose $\mathrm{B}_{1-6-12} ; \mathrm{P}+\mathrm{MB}$ - cisplatin + medium-dose $\mathrm{B}_{1-6-12} ; \mathrm{P}+\mathrm{HB}-$ cisplatin + high-dose $\mathrm{B}_{1-6-12} ;$ ${ }^{*} p<0.01 \mathrm{P}$ vs. $C$ and $\mathrm{P}+\mathrm{HB} ; \mathrm{p}<0.05 \mathrm{P}$ vs. $\mathrm{P}+\mathrm{MB} ; \mathrm{p}<0.05$ $\mathrm{P}+\mathrm{LB}$ vs. $\mathrm{C}$ and $\mathrm{P}+\mathrm{HB}$.

the cisplatin $+M B$ were not significantly different from those of the control groups. No significant changes were observed between groups in other parameters. However, there were trends toward thinner myelin sheath in all cisplatin-treated groups and higher number of fibres in the cisplatin and cisplatin + HB groups.

\section{DRG morphometry}

At the fifth week, the number of DRG neurons and nuclear area were significantly decreased in the cisplatin compared with the control groups (Table 2). However, the nucleolar area was significantly decreased in all cisplatin-treated compared with the control groups. All cisplatin $+B_{1-6-12}$ groups had values between those of the control and cisplatin groups.

\section{Transmission electron microscopic analysis}

Separation between the endothelial cell and pericyte or pericyte detachment appeared to be wider and more frequent in the nerve and DRG capillaries from the cisplatin compared with the control groups (Fig. 2). The detachment was less prominent in the cisplatin $+B_{1-6-12}$ groups. When the number of capillaries with detachment was compared with that of total capillaries included, the ratio was significantly higher in the cisplatin than the control groups (Fig. 3). All doses of $B_{1-6-12}$ had the significantly lower ratio than the cisplatin group but remained higher than the control group. However, when considering data of both sciatic nerve and DRG, the ratio of the cisplatin $+\mathrm{MB}$ group was the least different from that of the 
Table 1. Nerve morphometry

\begin{tabular}{lccccc}
\hline Group & Fibre diameter $[\mu \mathrm{m}]$ & Myelin thickness $[\mu \mathrm{m}]$ & g ratio & Fibre density $\left[/ \mu \mathrm{m}^{2}\right]$ & Number of fibre \\
\hline Control (C) & $6.52 \pm 0.14$ & $1.29 \pm 0.11$ & $0.61 \pm 0.03$ & $14,583.3 \pm 557.7$ & $8,798 \pm 295$ \\
Cisplatin (P) & $5.57 \pm 0.09^{\mathrm{a}}$ & $1.21 \pm 0.02$ & $0.57 \pm 0.01$ & $20,305.6 \pm 1,073.8^{\mathrm{b}}$ & $10,026 \pm 127$ \\
Cisplatin + LB & $5.77 \pm 0.11^{\mathrm{b}}$ & $1.21 \pm 0.02$ & $0.58 \pm 0.01$ & $18,437.5 \pm 681.8$ & $8,680 \pm 596$ \\
Cisplatin + MB & $6.62 \pm 0.12$ & $1.14 \pm 0.11$ & $0.63 \pm 0.02$ & $13,555.6 \pm 944.6$ & $8,742 \pm 266$ \\
Cisplatin + HB & $5.55 \pm 0.23^{\mathrm{a}}$ & $1.04 \pm 0.04$ & $0.62 \pm 0.00$ & $22,333.3 \pm 1,686.1^{\mathrm{a}}$ & $9,673 \pm 244$ \\
\hline
\end{tabular}

Data are means \pm standard error of mean; ${ }^{a} p<0.01$ vs. $C$ and $M B$, ${ }^{b} p<0.05$ vs. $C$ and $M B ; L B-$ low-dose $B_{1-6-12} ; M B-$ medium-dose $B_{1-6-12} ; H B-$ high-dose $B_{1-6-12}$

Table 2. Dorsal root ganglion morphometry

\begin{tabular}{|c|c|c|c|}
\hline Group & No. of neuron & Nuclear area $\left[\mu \mathrm{m}^{2}\right]$ & Nucleolar area $\left[\mu \mathrm{m}^{2}\right]$ \\
\hline Control (C) & $21,170 \pm 682$ & $150.43 \pm 5.64$ & $14.28 \pm 0.42$ \\
\hline Cisplatin (P) & $15,581 \pm 328^{a}$ & $128.18 \pm 4.73^{b}$ & $10.29 \pm 0.62^{c}$ \\
\hline Cisplatin + LB & $18,113 \pm 511$ & $133.01 \pm 4.67$ & $11.40 \pm 0.46^{d}$ \\
\hline Cisplatin + MB & $19,024 \pm 766$ & $137.79 \pm 5.46$ & $11.27 \pm 0.14^{d}$ \\
\hline Cisplatin + HB & $19,730 \pm 1,024$ & $130.26 \pm 4.93$ & $11.16 \pm 0.53^{d}$ \\
\hline
\end{tabular}

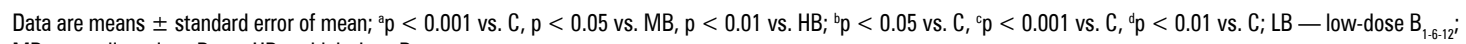
$\mathrm{MB}$ - medium-dose $\mathrm{B}_{1-6-12} ; \mathrm{HB}$ - high-dose $\mathrm{B}_{1-6-12}$

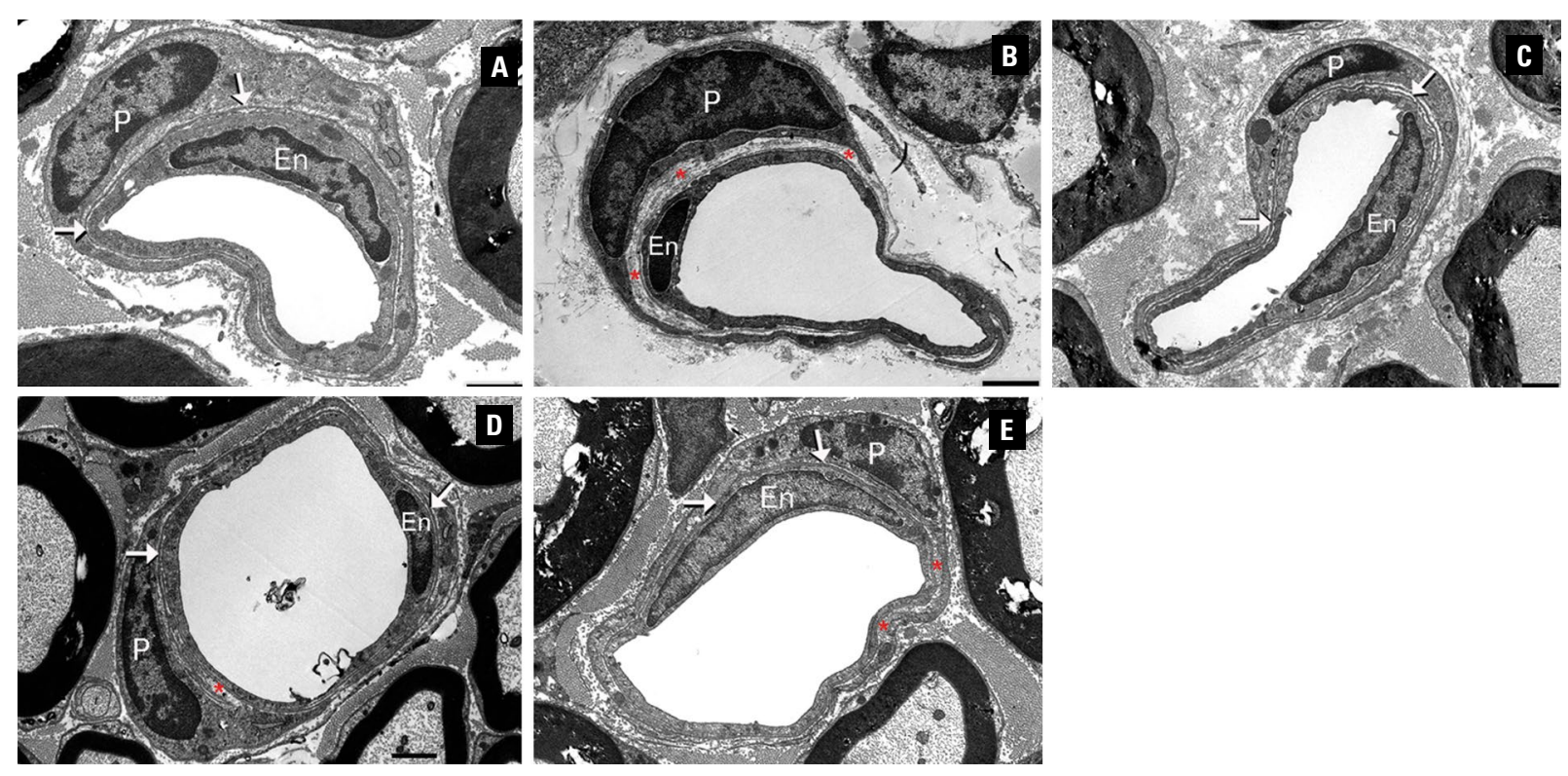

Figure 2. Representative ultrastructural images of capillaries in the sciatic nerves from the control (A), cisplatin (B), cisplatin + low-dose $B_{1-6-12}(\mathbf{C})$, cisplatin + medium-dose $B_{1-6-12}$ (D) and cisplatin + high-dose $B_{1-6-12}$ (E). Arrows indicate the basement membrane shared between the endothelial cell (En) and pericyte (P); ${ }^{*}$ pericyte detachment or separation between the endothelial cells and pericytes; scale bars $=1 \mu \mathrm{m}$.

control group. The changes in the proximal and distal parts of nerve were similar.

The separation distance of the cisplatin group was significantly longer than that of the control group in the sciatic nerves but not the DRG (Fig. 4). All cisplatin $+B_{1-6-12}$ groups had shorter distances compared with the cisplatin group. The values of the cisplatin $+\mathrm{MB}$ group were the closest to those of the control group. It is worth mentioning that only the cisplatin $+\mathrm{HB}$ group had significantly longer distance than the control group in the DRG. As for the thickness of basement membrane at the separation, there were no significant differences between groups (data not shown). Other pathological findings such as accumulation of lysosomes or vacuoles were not detected in the pericytes as well as endothelial cells in any group. 


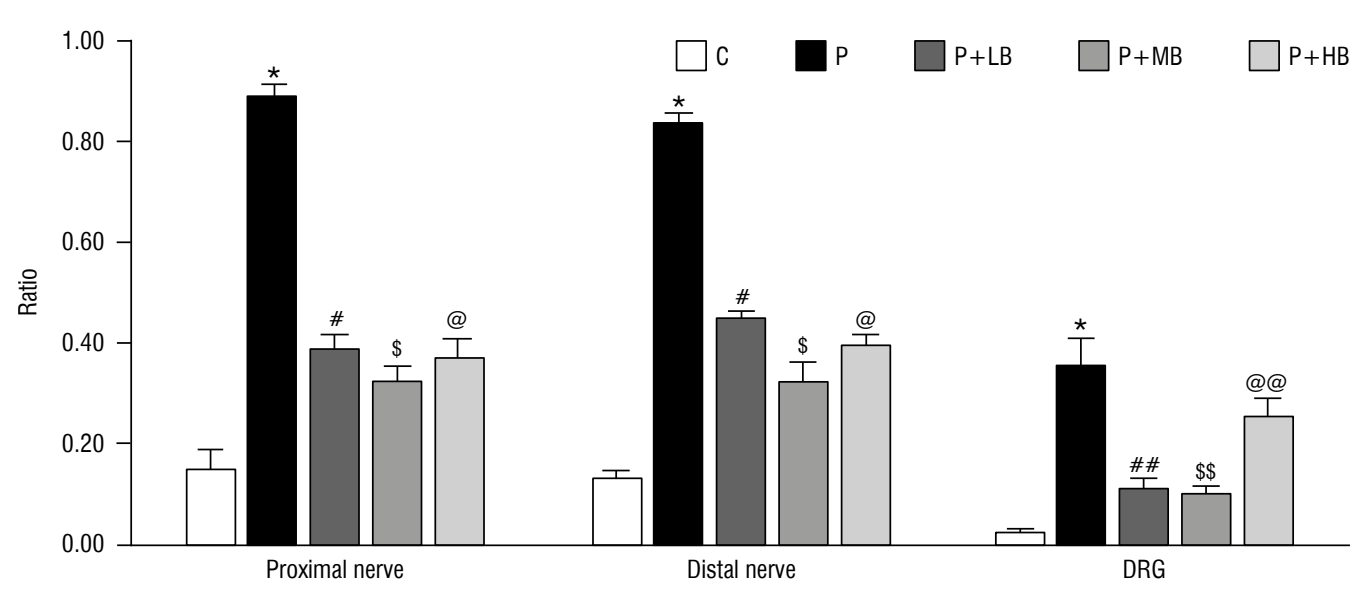

Figure 3. Ratio of the number of capillaries with pericyte detachment from endothelial cells to the total number of capillaries examined in the proximal and distal parts of sciatic nerve including L5 dorsal root ganglion (DRG). The graph shows means and standard error of mean; $\mathrm{C}$ - control; $\mathrm{P}$ - cisplatin; $\mathrm{P}+\mathrm{LB}-$ cisplatin + low-dose $\mathrm{B}_{1-6-12^{\prime}} \mathrm{P}+\mathrm{MB}-$ cisplatin + medium-dose $\mathrm{B}_{1-6-12} ; \mathrm{P}+\mathrm{HB}-$ cisplatin + high-dose $B_{1-6-12} ;{ }^{*} p<0.001 P$ vs. $C, \# p<0.001 P+L B$ vs. $P$ and $p<0.001 P+L B$ vs. $C ; \# \# p<0.001 P+L B$ vs. $P ; \$ p<0.001 P+M B$ vs. $P$ and $p<0.01 P+M B$ vs. $C ; \$ p<0.001 P+M B$ vs. $P ; @ p<0.001 P+H B$ vs. $P$ and $p<0.01 P+H B$ vs. C; $@ @ p<0.01 P+H B$ vs. C.

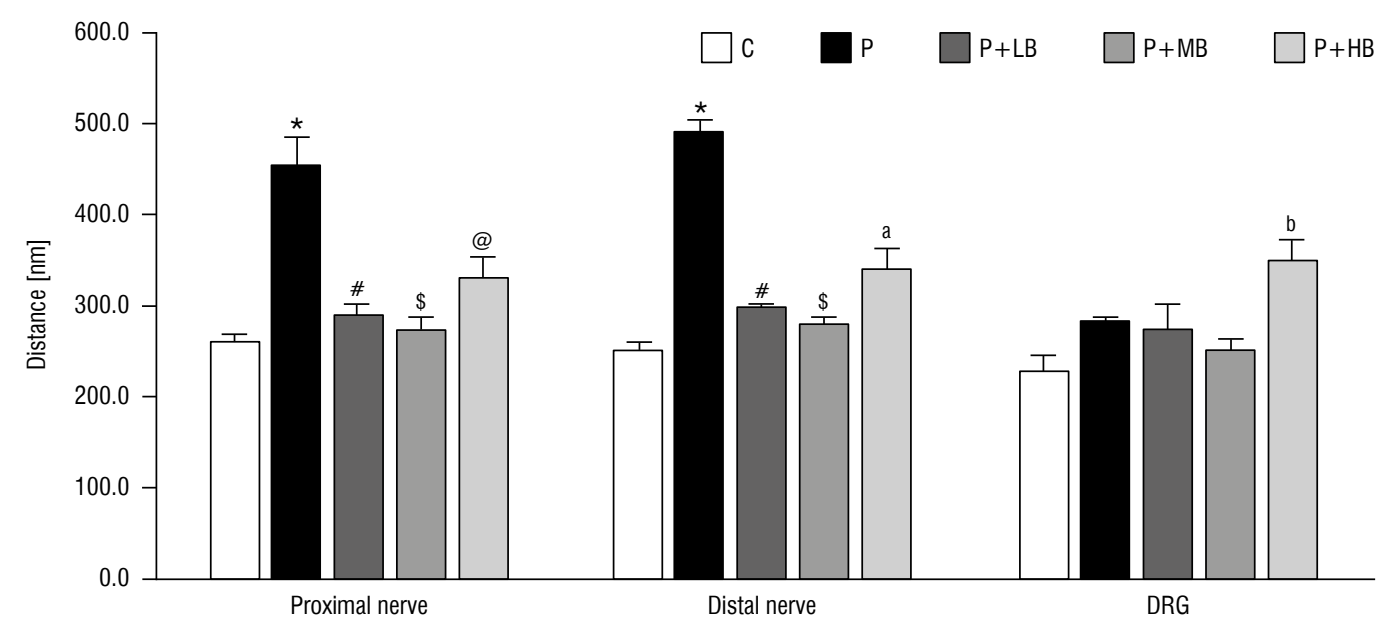

Figure 4. Distance at the widest separation between the endothelial cells and pericytes in the proximal and distal parts of sciatic nerve including $L 5$ dorsal root ganglion (DRG). The graph shows means and standard error of mean; $C$ - control; $P$ - cisplatin; $P+L B-$ cisplatin + low-dose $\mathrm{B}_{1-6-12} ; \mathrm{P}+\mathrm{MB}$ - cisplatin + medium-dose $\mathrm{B}_{1-6-12} ; \mathrm{P}+\mathrm{HB}$ - cisplatin + high-dose $\mathrm{B}_{1-6-12} ;{ }^{*} \mathrm{p}<0.001 \mathrm{P}$ vs. $\mathrm{C} ; \# \mathrm{p}<0.001$ $\mathrm{P}+\mathrm{LB}$ vs. $\mathrm{P} ; \mathrm{\$ p}<0.001 \mathrm{P}+\mathrm{MB}$ vs. $\mathrm{P} ; @ \mathrm{p}<0.001 \mathrm{P}+\mathrm{HB}$ vs. $\mathrm{P} ;{ }^{a} \mathrm{p}<0.001 \mathrm{P}+\mathrm{HB}$ vs. $\mathrm{P}$ and $\mathrm{p}<0.01 \mathrm{P}+\mathrm{HB}$ vs. $\mathrm{C} ;{ }^{b} p<0.05 \mathrm{P}+\mathrm{HB}$ vs. $\mathrm{C}$.

\section{Cell viability and caspase-3 activity}

Cell viability of the HUVEC was significantly reduced in the cisplatin group compared with the control group (Fig. 5). This was in agreement with the increased caspase-3 activity after cisplatin treatment. Similarly, cisplatin treatment in the HBVP resulted in lower viability and higher caspase-3 activity (Fig. 5). $B_{1-6-12}$ was able to significantly enhance the viability of both HUVEC and HBVP but yielded only trends toward less caspase-3 activity in both cell types.

\section{TEER}

Transendothelial electrical resistance of the cisplatin group was significantly lower than that of the control group (Fig. 6). Moreover, concomitant addition of $\mathrm{B}_{1-6-12}$ with cisplatin caused partial restoration of the resistance.

\section{Expression of tight junction proteins}

Expression of occludin and zonula occluden-2 (ZO-2) was significantly decreased in the HUVEC exposed to cisplatin compared with the controls (Fig. 7). However, the expression of claudin-5 and zonula occluden-1 (ZO-1) was not significantly different. Following $B_{1-6-12}$ treatment, the expression of occludin and ZO-2 was completely reversed to those seen in the control group. It is worth mentioning that the ZO-1 expression was significantly enhanced in the cisplatin $+B_{1-6-12}$ group compared with the cisplatin group. 

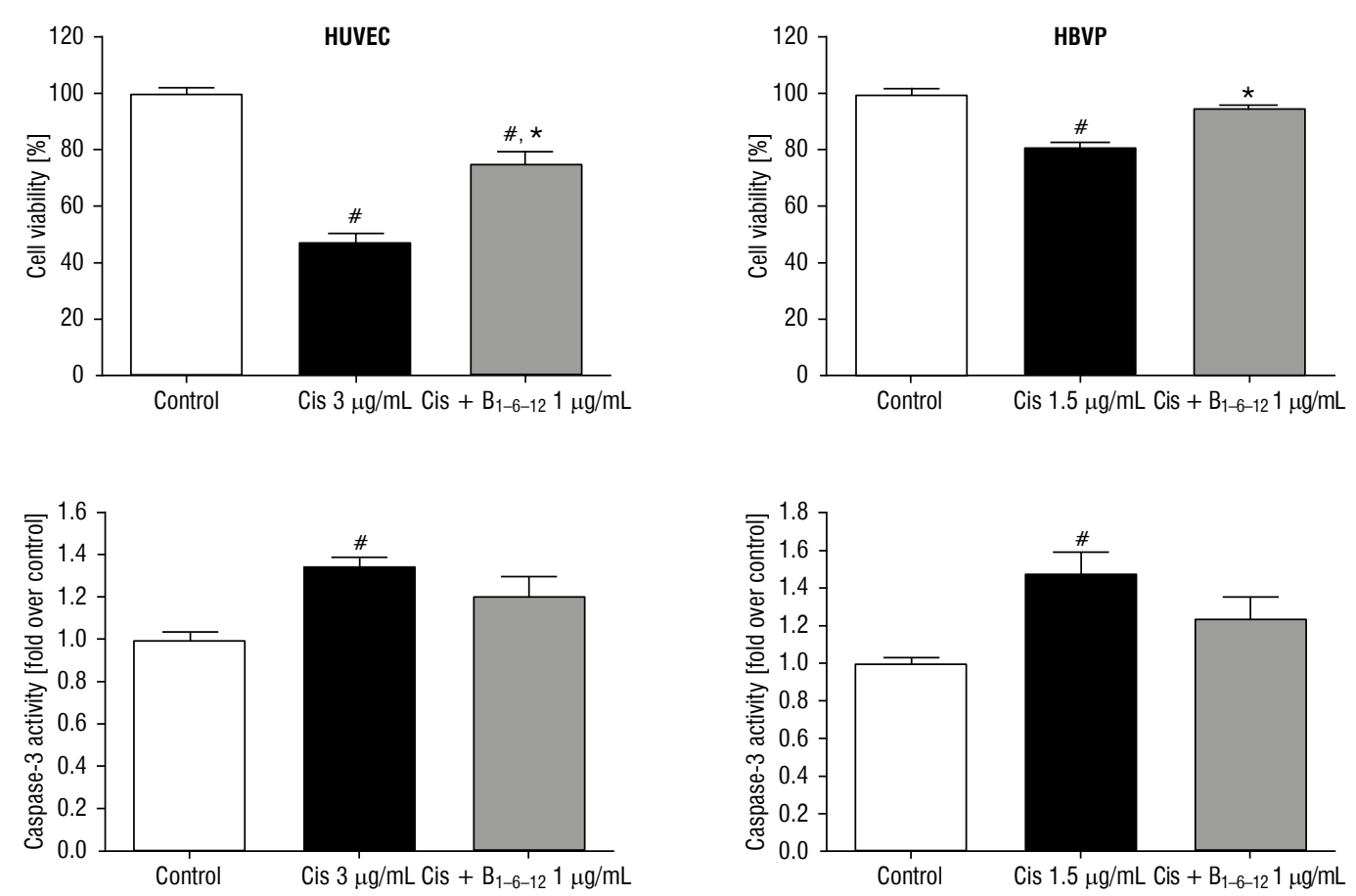

Figure 5. Cell viability and caspase-3 activity of human umbilical vein endothelial cell (HUVEC, left column) and human brain vascular pericyte (HBVP, right column). The graphs show means and standard error of mean; Cis - cisplatin; $\# p<0.01$ vs. control group; ${ }^{*} p<0.01$ vs. cisplatin group.

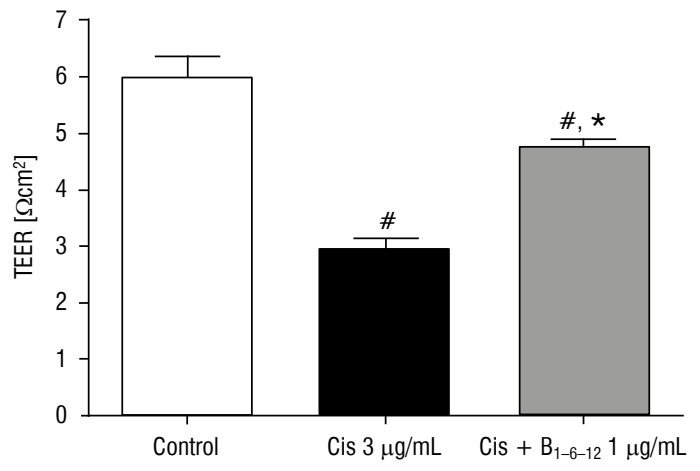

Figure 6. Transendothelial electrical resistance (TEER) of human umbilical vein endothelial cell. The graph shows means and standard error of mean; Cis - cisplatin; \#p $<0.01$ vs. control group; ${ }^{*} \mathrm{p}<0.01$ vs. cisplatin group.

\section{DISCUSSION}

The rats treated with cisplatin developed neuropathy characterised by thermal hypoalgesia and morphometric changes: reduced fibre diameter, increased fibre density, loss of DRG neurons and shrinkage of nucleus and nucleolus. These features of cisplatin-induced neuropathy were similar to those previously reported [1, 2, 28, 29]. Higher density of nerve fibres was likely due to shrinkage of fibres and slight increase in the number of fibres. Several of these functional and pathological abnormalities were com- parable to those observed in cancer patients treated with cisplatin $[23,27]$.

Vitamin B1, B6 and B12 had beneficial effects on thermal sensation and morphometry. High and medium, but not low doses of $B_{1-6-12}$ could significantly reduce the prolonged thermal latency seen in the cisplatin group. Furthermore, medium dose was better than low and high doses in restoring the fibre diameter and fibre density toward controls. As for DRG, all doses of $B_{1-6-12}$ appeared to have modest effects on the loss of neurons including shrinkage of nucleus and nucleolus caused by cisplatin. It is worth noting that $B_{1-6-12}$ did not have any significant effect on the weight loss used to indicate the general toxicity of cisplatin.

The previous study has demonstrated the pericyte detachment from endothelial cells in the nerves from cisplatin-treated rats which was rarely seen in the controls [12]. This study confirmed with quantitative analysis that pericytes detached with significantly higher frequencies and severity in the cisplatin than in the control rats. It is worth mentioning that despite the higher frequencies of detachment in the DRG from all cisplatin-treated compared with the control groups, the distances were not significantly different. More research is required to clarify why the pericytes detach with shorter distances in the DRG than in the nerves. 

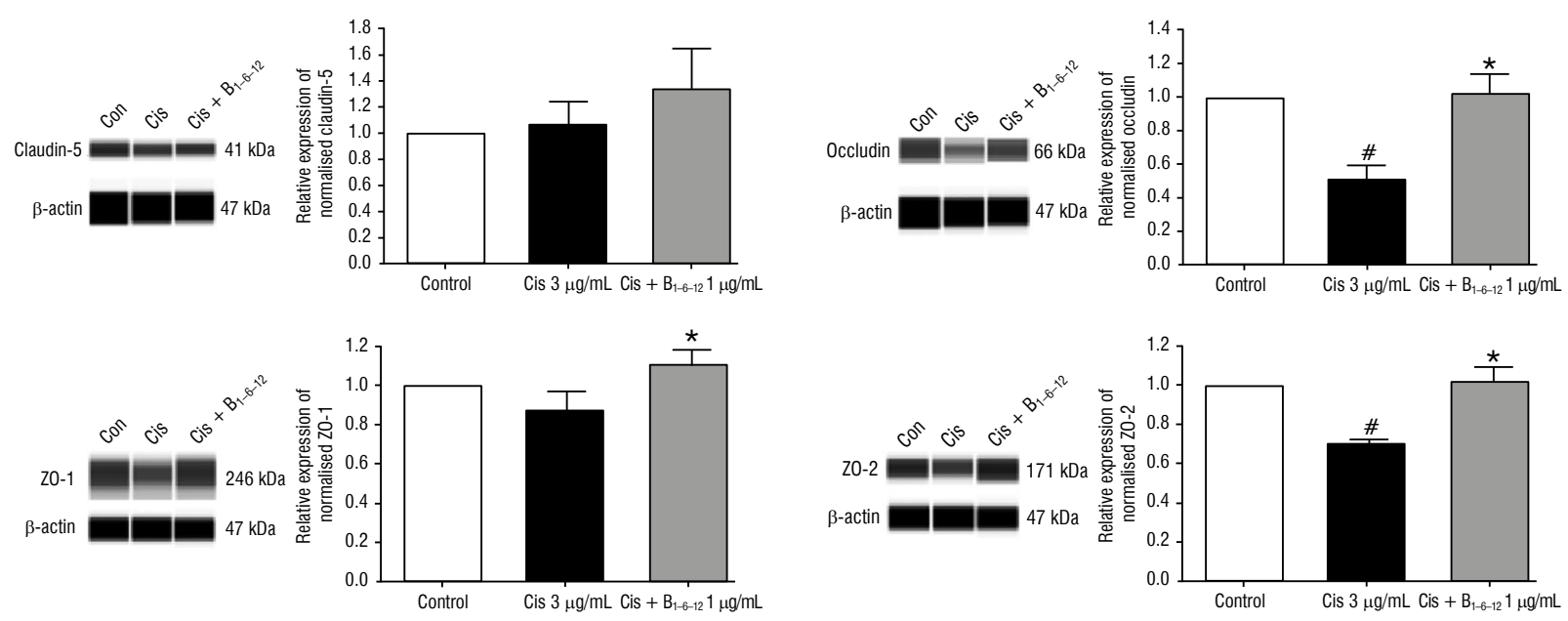

Figure 7. Protein expression of claudin-5, occludin, zonula occluden-1 and -2 (ZO-1 and ZO-2) representative immunoblots are shown. The density of each protein was normalised to that of $\beta$-actin. The graphs show means and standard error of mean; Con - control; Cis - cisplatin; $\# p<0.05$ vs. control group; ${ }^{*} p<0.05$ vs. cisplatin group.

Pericyte detachment or migration has been shown in various conditions and organs. Increased migration of pericytes was observed in the retina of diabetic rats [21]. More pericytes in the anterior pituitary gland detached from the capillary walls of the prolactinoma rats than the normal controls [11]. Pericytes migrated from the vascular wall in response to traumatic brain injury [7]. Implications of the pericyte detachment are still unclear. However, since pericytes, endothelial cells and vascular basement membrane co-operate as the BNB to regulate the microvascular functions [20], the detachment is likely deleterious to the nerve. At least in the cisplatin-induced ototoxicity model, ultrastructural changes in the endothelial cells and pericyte migration in the stria vascularis of cochlea were associated with auditory impairment [30].

All doses of $B_{1-6-12}$ could alleviate the elevation in cisplatin-induced pericyte detachment in both sciatic nerves and DRG with the best result seen in the medium-dose group. Moreover, both low and medium doses of $B_{1-6-12}$ could equally normalise the distances of detachment. Nevertheless, high dose of $B_{1-6-12}$ may be less favourable or even harmful, especially in the DRG. The detachment distance in the high-dose group was significantly longer than those in the other groups. This dose-dependent effect of $B_{1-6-12}$ will be discussed later.

Due to the effects of cisplatin and $B_{1-6-12}$ on the nerve and DRG capillaries described above, the question which cell component of the BNB was affected by the drugs has emerged. This was clarified using separate cultures of endothelial cells (HUVEC) and pericytes (HBVP). Cisplatin reduced the viability of both cell types at least via activation of caspase-3. This was in consistent with the previous reports of endothelial cell apoptosis and enhanced caspase-3 activity following cisplatin treatment $[8,9,15,19]$. Furthermore, TEER which represents the barrier function, was also reduced by cisplatin. Moreover, the expression of tight junction proteins in the HUVEC was examined. Expression of occludin and ZO-2, but not claudin-5 and ZO-1, was significantly lower in the cisplatin-treated cells compared with the controls. Reduced expression of tight junction proteins was also found in the stria vascularis of cochlea in cisplatin-treated mice [30].

Pericytes as well as endothelial cells are important for the normal functions of BNB and damage in any of these components might cause neuropathy. The above data from cell culture and ultrastructural analysis indicate that cisplatin causes endothelial and pericyte damage including the BNB disruption. The previous studies have already suggested the importance of tight junction proteins in the BNB integrity and nerve functions. Reduced level of claudin-5 was associated with BNB dysfunction in chronic inflammatory demyelinating polyradiculoneuropathy (CIDP) [14]. Shimizu et al. [25] showed that pericytes controlled the expression of claudin- 5 in the endothelial cells through secretion of growth factors. Besides claudin-5, nerve pericytes also express other important components of the BNB, for example, fibronectin, collagen type IV [26].

Vitamin B1, B6 and B12 could partially correct the reduced viability of both HUVEC and HBVP after 
cisplatin administration. Furthermore, the decreased TEER was also alleviated by $B_{1-6-12}$. Regarding the tight junction proteins, $B_{1-6-12}$ was able to correct the reduced expression of occludin and ZO- 2 and further enhanced the expression of ZO-1 above the control level. All these data suggest that beneficial effects of $B_{1-6-12}$ on functional and morphometric parameters of cisplatin neurotoxicity described earlier might be exerted at least via improvement in the BNB functions. This could be due to less cell toxicity and enhanced expression of some tight junction proteins. Although numerous agents targeting various mechanisms were effective in experimental cisplatin neuropathy, they failed to show significant benefits in clinical trials [24]. Vitamin B1, B6 and B12 are water-soluble vitamins essential for normal functions of the nervous system and frequently prescribed for neuropathies from various causes. However, current evidence of efficacy of B vitamins in chemotherapy-induced neuropathy is still inconclusive [18]. The results of this study support the continued effort to develop $B_{1-6-12}$ as the potential treatment for cisplatin-induced neuropathy. It is worth noting that less or unfavourable effects of high-dose $B_{1-6-12}$ in the morphometric analysis of DRG and sciatic nerves were found in this study. This might be due to toxicity of all or specific $B$ vitamins. Excessive intake of pyridoxine $\left(B_{6}\right)$ can cause neuropathy [10]. Therefore, optimal dose of these $B$ vitamins must be determined to prevent the overdose side effects. Data in this study suggest the medium dose $(300 \mathrm{mg} / \mathrm{kg} /$ day per oral) of $B_{1-6-12}$ as the most suitable.

The results in this study also suggest the BNB impairment as additional important mechanism underlying cisplatin-induced neuropathy. This is in accordance with the previous study showing the BNB abnormalities in CIDP cases [14]. However, it remains to be proved whether these alterations in the BNB occur in the patients with cisplatin neuropathy. Moreover, the BNB integrity should be examined in neuropathies from other chemotherapeutic drugs or other causes. In the future, drugs with beneficial effects on endothelial cells or pericytes can be assessed for potential treatments against peripheral neuropathy with impaired BNB.

\section{CONCLUSIONS}

This study has demonstrated the favourable effects of $B_{1-6-12}$ on thermal hypoalgesia and abnormal morphometric parameters of the sciatic nerves and DRG induced by cisplatin. Ultrastructural analysis revealed that cisplatin stimulated pericyte detachment in the capillaries in those tissues. In addition, cell culture experiments showed reduced viability of endothelial cells and pericytes, transendothelial electrical resistance and expression of some tight junction proteins. $B_{1-6-12}$ especially the medium dose, could improve the sensory deficit and structural alterations. Moreover, cell viability, barrier function and tight junction proteins were also corrected by $\mathrm{B}_{1-6-12}$. These data suggest that BNB disruption is one of the pathological mechanisms underlying cisplatin-induced neuropathy and $B_{1-6-12}$ are the potential treatment.

\section{Acknowledgements}

We are grateful to the Research Affairs, Faculty of Medicine, Chulalongkorn University for assistance regarding transmission electron microscopy. This work was supported by the Faculty of Medicine, Chulalongkorn University under the Ratchadaphiseksomphot Fund (RA62/022 \& RA63/030) and the $90^{\text {th }}$ Anniversary of Chulalongkorn University Fund (Ratchadaphiseksomphot Endowment Fund, GCUGR1125631039D-39).

\section{Conflict of interest: None declared}

\section{REFERENCES}

1. Al Moundhri MS, Al-Salam S, Al Mahrouqee A, et al. The effect of curcumin on oxaliplatin and cisplatin neurotoxicity in rats: some behavioral, biochemical, and histopathological studies. J Med Toxicol. 2013; 9(1): 25-33, doi: 10.1007/ s13181-012-0239-x, indexed in Pubmed: 22648527.

2. Authier N, Gillet JP, Fialip J, et al. An animal model of nociceptive peripheral neuropathy following repeated cisplatin injections. Exp Neurol. 2003; 182(1): 12-20, doi: 10.1016/s0014-4886(03)00003-7, indexed in Pubmed: 12821373.

3. Bartoszyk GD, Wild A. B-vitamins potentiate the antinociceptive effect of diclofenac in carrageenin-induced hyperalgesia in the rat tail pressure test. Neurosci Lett. 1989; 101(1): 95-100, doi: 10.1016/0304-3940(89)90447-3, indexed in Pubmed: 2771158.

4. Chentanez V, Cha-oumphol P, Kaewsema A, et al. Accuracy of the three-window sampling method in morphometric analysis of human sural nerve. J Neurosci Methods. 2006; 157(1): 154-157, doi: 10.1016/j.jneumeth.2006.04.001, indexed in Pubmed: 16704879.

5. Dieckmann KP, Struss WJ, Budde U. Evidence for acute vascular toxicity of cisplatin-based chemotherapy in patients with germ cell tumour. Anticancer Res. 2011; 31(12): 4501-4505, indexed in Pubmed: 22199322.

6. Dilruba S, Kalayda GV. Platinum-based drugs: past, present and future. Cancer Chemother Pharmacol. 2016; 77(6): 1103-1124, doi: 10.1007/s00280-016-2976-z, indexed in Pubmed: 26886018. 
7. Dore-Duffy P, Owen C, Balabanov R, et al. Pericyte migration from the vascular wall in response to traumatic brain injury. Microvasc Res. 2000; 60(1): 55-69, doi: 10.1006/ mvre.2000.2244, indexed in Pubmed: 10873515.

8. Dursun $B, H e Z$, Somerset $H$, et al. Caspases and calpain are independent mediators of cisplatin-induced endothelial cell necrosis. Am J Physiol Renal Physiol. 2006; 291(3): F578-F587, doi: 10.1152/ajprenal.00455.2005, indexed in Pubmed: 16622172.

9. Eguchi R, Fujimori $Y$, Ohta $T$, et al. Calpain is involved in cisplatin-induced endothelial injury in an in vitro three-dimensional blood vessel model. Int J Oncol. 2010; 37(5): 1289-1296, doi: 10.3892/ijo_00000780.

10. Hadtstein F, Vrolijk M. Vitamin B-6-induced neuropathy: exploring the mechanisms of pyridoxine toxicity. Adv Nutr. 2021; 12(5): 1911-1929, doi: 10.1093/advances/ nmab033, indexed in Pubmed: 33912895.

11. Jindatip D, Fujiwara K, Sarachana T, et al. Characteristics of pericytes in diethylstilbestrol (DES)-induced pituitary prolactinoma in rats. Med Mol Morphol. 2018; 51(3): 147-155, doi: 10.1007/s00795-018-0180-4, indexed in Pubmed: 29344720.

12. Jindatip D, Nopparat $W$, Kobutree $P$, et al. Pericyte loss and detachment in experimental cisplatin-induced neuropathy. Int J Morphol. 2019; 37(2): 509-514, doi: 10.4067/s071795022019000200509.

13. Jolivalt CG, Mizisin LM, Nelson A, et al. B vitamins alleviate indices of neuropathic pain in diabetic rats. Eur J Pharmacol. 2009; 612(1-3): 41-47, doi: 10.1016/j. ejphar.2009.04.028, indexed in Pubmed: 19393643.

14. Kanda T, Numata $Y$, Mizusawa H. Chronic inflammatory demyelinating polyneuropathy: decreased claudin- 5 and relocated ZO-1. J Neurol Neurosurg Psychiatry. 2004; 75(5): 765-769, doi: 10.1136/jnnp.2003.025692, indexed in Pubmed: 15090575.

15. Kirchmair R, Walter DH, li M, et al. Antiangiogenesis mediates cisplatin-induced peripheral neuropathy: attenuation or reversal by local vascular endothelial growth factor gene therapy without augmenting tumor growth. Circulation. 2005; 111(20): 2662-2670, doi: 10.1161/CIRCULATIONAHA.104.470849, indexed in Pubmed: 15897348.

16. Krarup-Hansen A, Helweg-Larsen S, Schmalbruch $H$, et al. Neuronal involvement in cisplatin neuropathy: prospective clinical and neurophysiological studies. Brain. 2007; 130(Pt 4): 1076-1088, doi: 10.1093/brain/awl356, indexed in Pubmed: 17301082.

17. Li SH, Chen WH, Tang Y, et al. Incidence of ischemic stroke post-chemotherapy: a retrospective review of 10,963 patients. Clin Neurol Neurosurg. 2006; 108(2): 150-156, doi: 10.1016/j.clineuro.2005.03.008, indexed in Pubmed: 16412836.

18. Liu YW, Liu CT, Su YL, et al. A narrative review of complementary nutritional supplements for chemotherapy-induced peripheral neuropathy. Altern Ther Health Med. 2020; 26(4): 43-49, indexed in Pubmed: 31634876.
19. Nuver J, De Haas EC, Van Zweeden M, et al. Vascular damage in testicular cancer patients: A study on endothelial activation by bleomycin and cisplatin in vitro. Oncol Rep. 2009; 23(1), doi: 10.3892/or_00000630.

20. Peltonen $S$, Alanne $M$, Peltonen J. Barriers of the peripheral nerve. Tissue Barriers. 2013; 1(3): e24956, doi: 10.4161/ tisb.24956, indexed in Pubmed: 24665400.

21. Pfister $F$, Feng $Y$, vom Hagen $F$, et al. Pericyte migration: a novel mechanism of pericyte loss in experimental diabetic retinopathy. Diabetes. 2008; 57(9): 2495-2502, doi: 10.2337/db08-0325, indexed in Pubmed: 18559662.

22. Quasthoff S, Hartung $\mathrm{H}$. Chemotherapy-induced peripheral neuropathy. J Neurol. 2002; 249(1): 9-17, doi: 10.1007/ pl00007853.

23. Roelofs RI, Hrushesky W, Rogin J, et al. Peripheral sensory neuropathy and cisplatin chemotherapy. Neurology. 1984; 34(7): 934-938, doi: 10.1212/wnl.34.7.934, indexed in Pubmed: 6330613.

24. Santos NA, Ferreira RS, Santos AC. Overview of cisplatin-induced neurotoxicity and ototoxicity, and the protective agents. Food Chem Toxicol. 2020; 136: 111079, doi: 10.1016/j.fct.2019.111079, indexed in Pubmed: 31891754.

25. Shimizu F, Sano Y, Abe MA, et al. Peripheral nerve pericytes modify the blood-nerve barrier function and tight junctional molecules through the secretion of various soluble factors. J Cell Physiol. 2011; 226(1): 255-266, doi: 10.1002/jcp.22337, indexed in Pubmed: 20665675.

26. Shimizu F, Sano $Y$, Haruki $H$, et al. Advanced glycation end-products induce basement membrane hypertrophy in endoneurial microvessels and disrupt the blood-nerve barrier by stimulating the release of TGF- $\beta$ and vascular endothelial growth factor (VEGF) by pericytes. Diabetologia. 2011; 54(6): 1517-1526, doi: 10.1007/s00125-0112107-7, indexed in Pubmed: 21409414.

27. Thompson SW, Davis LE, Kornfeld M, et al. Cisplatin neuropathy. Clinical, electrophysiologic, morphologic, and toxicologic studies. Cancer. 1984; 54(7): 1269-1275, doi: 10.1002/1097-0142(19841001)54:7<1269::aid-cncr2820540707>3.0.co;2-9, indexed in Pubmed: 6088023.

28. Wongtawatchai T, Agthong S, Kaewsema A, et al. Sex-related differences in cisplatin-induced neuropathy in rats. J Med Assoc Thai. 2009; 92(11): 1485-1491, indexed in Pubmed: 19938741.

29. Wongtawatchai T, Agthong S, Kaewsema A, et al. Altered phosphorylation of mitogen-activated protein kinases in dorsal root ganglia and sciatic nerve of rats with cisplatin-induced neuropathy. Asian Biomed (Res Rev News). 2012; 6: 397-411.

30. Zhang N, Cai J, Xu L, et al. Cisplatin-Induced stria vascularis damage is associated with inflammation and fibrosis. Neural Plast. 2020; 2020: 8851525, doi: 10.1155/2020/8851525, indexed in Pubmed: 33029120. 